\title{
CNT-enhanced carbon electrode for nitrite detection and water analysis
}

\begin{abstract}
In this contribution we present a novel electrode based on a bare pencil lead electrodes (PLEs) coated by carbon nanotubes. We investigate the sensitivity of the novel electrode during electrochemical analysis of aqueous solutions. For this purlose, a phosphate buffer having different nitrite concentrations has been subjected to meaurements with enhanced and bare PLEs over a wide potential range. The proposed coating increases the sensitivity to nitrite by increasing the electrode surface and its reactivity and shows therefore a significantly observable and sensitive signal.
\end{abstract}

Keywords: square wave voltammetry, nitrite, pencil electrode, carbon nanotubes

\section{Introduction}

Nitrite ions $\left(\mathrm{NO}_{2}^{-}\right)$can be found in food, water and soils [1]. In the food industry, nitrite is used in the curing of meat by preventing bacterial growth [2]. It is found as a natural component in vegetables and water due to chloramination [3]. High concentrations of nitrite ions are toxic to humans and animals. Especially during cooking, excess nitrite in meat can react with degradation products of amino acids to form carcinogenic nitrosamines [1, 4-6]. It also oxidizes hemoglobin to methemoglobin, which causes health problems like methemoglobinemia [7].

To detect harmful concentrations of nitrite in the food chain of human and animals, a reliable and cost-effective way of determining the concentration of nitrite in an aqueous solution is needed. The method of square wave voltammetry (SWV) provides excellent results in the quantitative analysis of chemical compounds in low concentrations [8]. The selective nature of this method is very useful to detect chemicals that are involved in electrochemical redox reactions. The sensitivity and the selectivity of the method can be further enhanced by specialized electrodes.

This enhancement is usually performed by coating a bulk electrode material within an electrocatalytic layer. Often used bulk materials for the electrodes are glassy carbon and pyrolytic carbon. These are expensive and are usually cleaned and re-used in several experiments. To evaluate a large amount of coatings in the optimization process a more cost-effective base electrode is needed.

In this work pencil lead electrodes (PLEs) are proposed as cheap and accessible base material for electrode prototyping. In an additional stage the electrodes are

Frank Wendler, Paul Aurosmit, Olfa Kanoun, Chair for Measurement and Sensor Technology, Chemnitz University of Technology, Reichenhainer Strasse 70, 09126 Chemnitz, Germany, e-mail: frank.wendler@etit.tu-chemnitz.de

https://doi.org/10.1515/9783110558920-014 
coated with multiwalled nanotubes (MWNTs) to enhance the sensitivity to the concentration of nitrite ions as they have a strong electrocatalytic property [9]. The aim of the paper is to invest the feasibility of this type of electrodes and to evaluate its sensitivity for measurements.

\section{Experimental}

\subsection{Solution preparation}

For the determination and graphical representation of the reaction kinetics, SWV was used.

SWV is known for well-defined current peaks at characteristic redox potentials [8]. It is also very sensitive to the electrode kinetics [8]. The redox reaction that takes place at the electrode is

$$
\mathrm{NO}_{2}^{-} \longleftrightarrow \mathrm{NO}_{2}+e^{-}
$$

A $0.65-\mathrm{M}(\mathrm{pH}=7)$ phosphate buffer solution $\left(\mathrm{NaH}_{2} \mathrm{PO}_{4} / \mathrm{Na}_{2} \mathrm{HPO}_{4}\right)$ from Grüssing $\mathrm{GmbH}$ and sodium nitrite of $99 \%$ purity was used to prepare the investigated solutions. The buffer solution was diluted to $0.325 \mathrm{M} ; 100 \mathrm{~mL}$ of the diluted buffer solution was contaminated with $0.7 \mathrm{mg}$ sodium nitrite and $100 \mathrm{~mL}$ of the buffer solution was left uncontaminated. Both base solutions were used to create samples with mixing ratios of $0 \%, 25 \%, 50 \%, 75 \%$ and $100 \%$. In this $100 \mathrm{~mL}$ of base solution the nitrite concentration was $100 \mu \mathrm{M}$. So in this investigation the different samples of $100 \%, 75 \%, 50 \%$, $25 \%$ and $0 \%$ correspond to concentrations of $100 \mu \mathrm{M}, 75 \mu \mathrm{M}, 50 \mu \mathrm{M}, 25 \mu \mathrm{M}$ and $0 \mu \mathrm{M}$.

\subsection{Preparation of electrodes}

The pencil lead consists of graphite, clay and wax has a resistance of approximately 6 Ohms. The whole pencil was heated to reduce the resistance and evaporate the excess binding material so that the properties of graphite are predominant. The pencil lead is covered by an insulating cover so that only the tip of the pencil is exposed to the solution.

For the enhancement, multiwalled carbon nanotubes (MWCNTs) of $2 \% \mathrm{w} / \mathrm{w}$ in 2-propanol (stabilized) were used from Future Carbon GmbH. The pencil lead was cleaned and then the tip was dipped in the prefabricated MWCNT solution.

The pencil lead was dried in a heater for 5 hours to evaporate the propanol and fixate the MWCNTs on the surface of the pencil lead. 


\subsection{Instrumentation and experimental setup}

A three-electrode cell was used for the electrochemical experiments. The measurements were conducted using the HEKA 310 potentiostat. The pencil lead was used as the working electrode. $\mathrm{Ag} / \mathrm{AgCl} 0.1 \mathrm{M} \mathrm{KCl}$ from $\mathrm{CH}$ Instruments, Inc. was used as the reference electrode. The counter electrode consisted of the platinum wire. The measurement setup is described in Fig. 1.

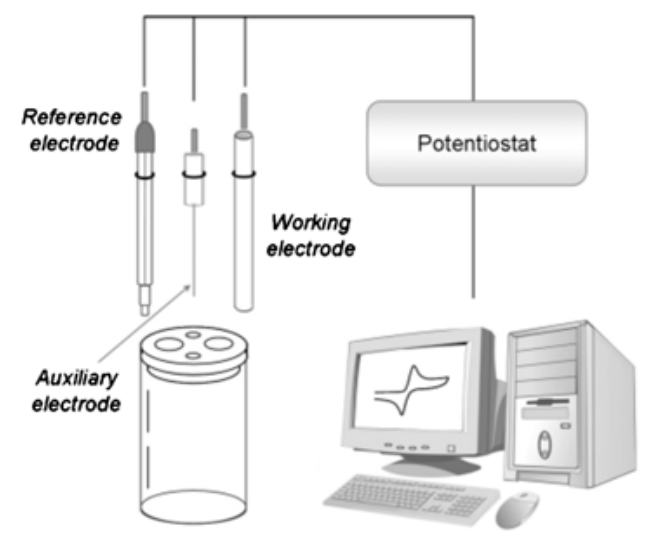

Fig. 1: Experimental setup [10].

\subsection{Instrument settings}

The start potential was set at $-0.5 \mathrm{~V}$ and the end potential at $1.2 \mathrm{~V}$. During the measurements in the negative potential range the samples were able to reach a steady state. The potential was increased in steps by $10 \mathrm{mV}$, and the square wave frequency was set to $1 \mathrm{~Hz}$. The scan rate was set at $10 \mathrm{mV} / \mathrm{s}$. The current was sampled from $98 \%-100 \%$ of the period so that the double layer capacitive effects can be avoided and only the Faradaic currents are represented in the data.

\section{Results and discussion}

The electrode was immersed in all five samples solutions and the SWV was conducted. The bare PLE in Fig. 2 shows a small current peak in the region of $850 \mathrm{mV}$. This peak is only visible at high concentrations of $100 \%$ and $75 \%$ of the base nitrite solution. We conclude that the bare PLE has a limited use in the detection of nitrite, but can be used as base electrode to observe the effects of enhancing coatings. 


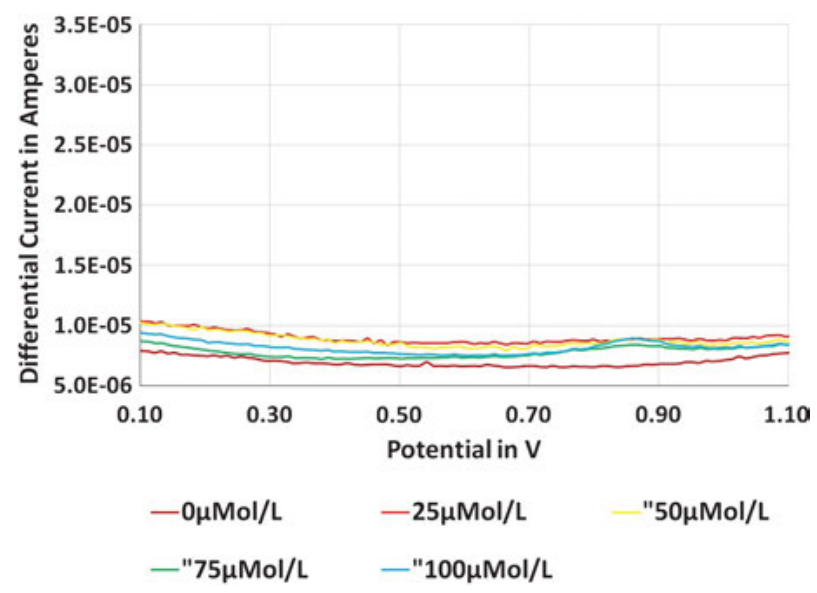

Fig. 2: SWV of pencil lead at different concentrations of nitrite.

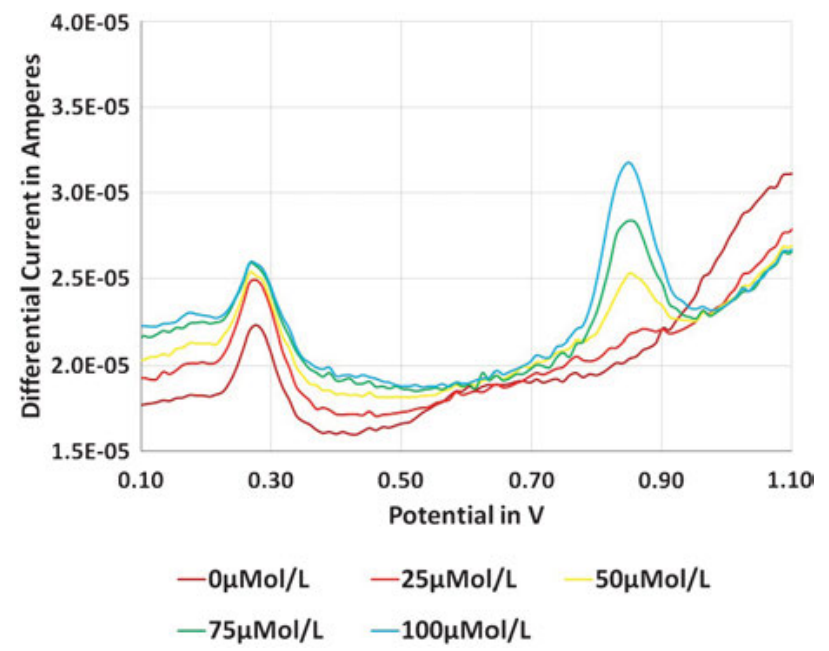

Fig. 3: SVW of CNT-coated PLE at different concentrations of nitrite.

The PLE was coated with the MWNT film and immersed in the same five sample solutions. The coated electrodes show an increased current at all concentrations as seen in Fig. 3 due to the increase in surface area of the nanomaterial coating. The current peak at $0.85 \mathrm{~V}$ for the nitrite is enhanced and also observable at lower concentrations of nitrite, as shown in Fig. 4. This increases the sensitivity and the detection limit for nitrites of this electrode type. Furthermore, a second peak can be observed at $0.275 \mathrm{~V}$. This peak has a constant level at all concentrations of nitrite. All the five samples always contain the same amount of phosphate buffer and de-ionized water irrespective of the nitrite concentration. So we conclude the peak at $0.275 \mathrm{~V}$ is related to the con- 


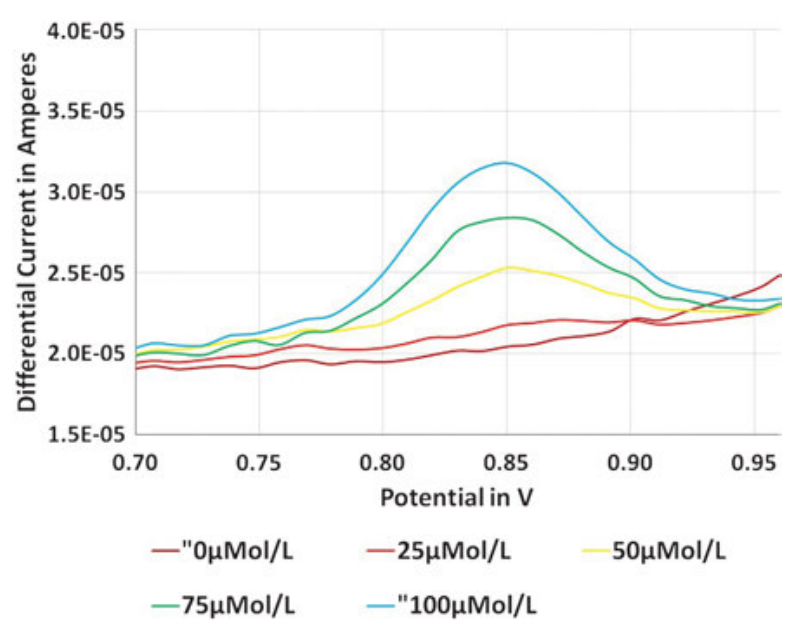

Fig. 4: SWV of CNT-PLE showing the peak currents at different concentrations of nitrite.

centration of the phosphate buffer. Since the SWV waveform for the bare PLE does not show any prominent peak at any potential, we assume that CNTs have a catalytic effect on the phosphate in the buffer solution.

The direct comparison between the voltammograms of the bare PLE and CNTenhanced PLE (CNT-PLE) for base solutions with concentrations of $0 \%$ and $100 \%$ of nitrite can be seen in Fig. 5 and Fig. 6, respectively. The peak related to the base solu-

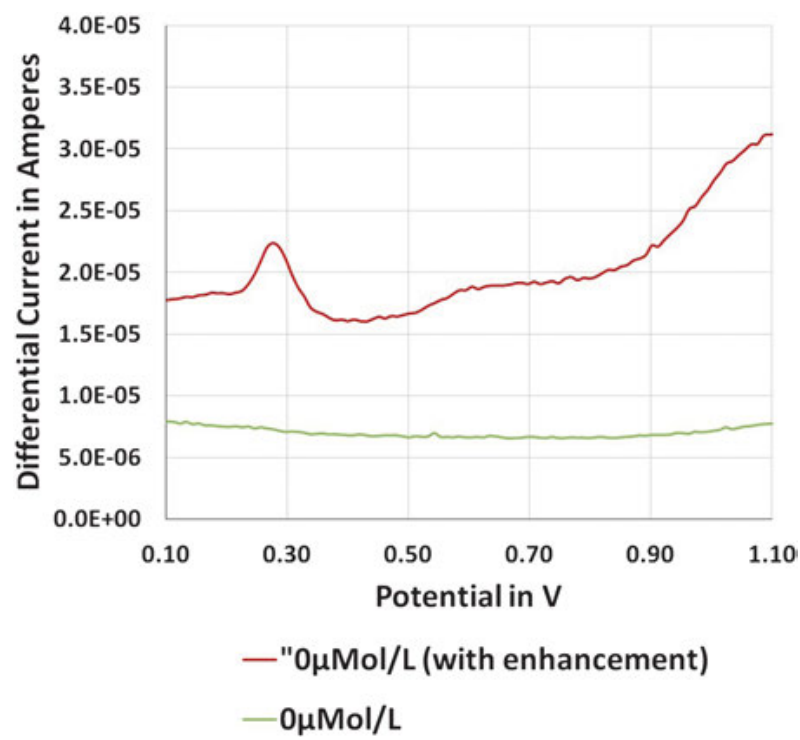

Fig. 5: SWV of PLE and CNT-PLE in $0 \%$ nitrite solution. 


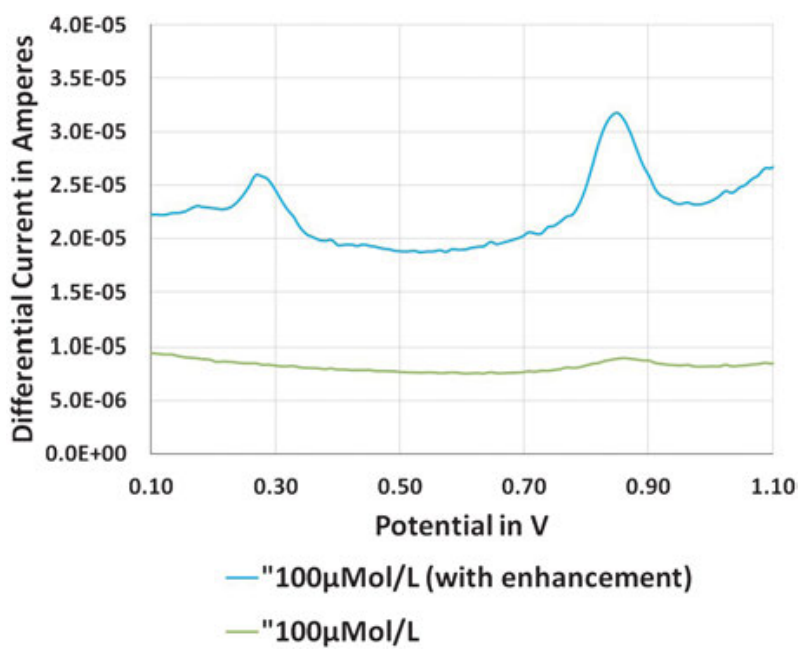

Fig. 6: SWV of PLE and CNT-PLE in $100 \%$ nitrite solution.

tion at $0.25 \mathrm{~V}$ is observable in both voltamogramms for the enhanced electrode. The nitrite peak at $0.85 \mathrm{~V}$ can be observed with both electrodes in solutions containing nitrite.

\section{Conclusion}

In this chapter, a novel CNT-coated PLE is proposed for nitrite measurements by SWV. The results show that the PLE can be used as an excellent base electrode for modification and evaluation. The modification by coating with CNTs show good results for concentrations of nitrite from $100 \mu \mathrm{M}$ to $25 \mu \mathrm{M}$.

The CNT-coated electrode can be made sensitive to other chemicals, like phosphate, to adapt to other chemicals and fields of application. The electrode material is cheap and can be enhanced with minimal effort. Therefore PLEs support a screening of large quantities of coating materials. For future work the miniaturization and optimization will be the main aim along with checking the sensitivity to other reagents, so that cheap and reliable electrochemical sensors can be created.

\section{Bibliography}

[1] Y. Wang, E. Laborda, and R. G. Compton, "Electrochemical oxidation of nitrite: Kinetic, mechanistic and analytical study by square wave voltammetry", Journal of Electroanalytical Chemistry, vol. 670, 2012. 
[2] E. F. Binkerd and O. E. Kolari, Food and Cosmetics Toxicology, vol. 13, no. 6, pp. 655-661, 1975.

[3] N. G. Hord, Y. Tang, and N. S. Bryan, The American Journal of Clinical Nutrition, vol. 90, no. 1, pp. 1-10, 2009.

[4] B. P. Kozub, N. V. Rees, and R. G. Compton, "Electrochemical determination of nitrite at a bare glassy carbon electrode; why chemically modify electrodes?”, Sensors and Actuators B, vol. 143, 2010.

[5] C. Yanping, Y. Changzhu, W. Zeng, M. Oyama, and P. U. Wenhong, “Electrochemical determination of nitrite using a gold nanoparticles-modified glassy carbon electrode prepared by the seed-mediated growth technique”, Journal Analytical Sciences, vol. 23, 2007.

[6] P. Manas and G. Vellaichamy, The Analyst, vol.135, pp. 2711-2716, 2020.

[7] L. Fu, Y. Shuhong, L. Thompson, and Y. Aimin, RSC Advances, vol. 5, pp. 40111-40116, 2015.

[8] F. M. Cubius, A. Ispas, A. Bund, and P. Ilea, "Square wave voltammetric detection of electroactive products resulting from electrochemical nitrate reduction in alkaline media", Journal of Electroanalytical Chemistry, vol. 675, 2012.

[9] S. Timur, U. Anik, D. Odaci, and Lo Gorton, "Development of a microbial biosensor based on carbon nanotube (CNT) modified electrodes", Electrochemistry Communications, vol. 9, no. 7, July 2007.

[10] A. Alvarez-Lueje, M. Perez, and C. Zapata, Pharmacology, Toxicology and Pharmaceutical Science, 1st edition, 978- 953-51-0099-7, 2012. 
